\title{
Vascular Spectrum of Imaging Findings in COVID-19: Ischemic, Hemorrhagic, and Thromboembolic Complications
} Pedro Neves Paiva de Castro, ${ }^{1,2} \odot$ Lucia Antunes Chagas, $^{1 \oplus}$ Eduardo Wajnberg, $^{1 \oplus}$ Roberto Santos, $^{1 \oplus}$ Pedro Cougo, $^{1 \oplus}$
Dequitier Carvalho Machado1

Américas Serviços Médicos, ${ }^{1}$ Rio de Janeiro, RJ - Brazil

Universidade do Estado do Rio de Janeiro (UERJ), ${ }^{2}$ Rio de Janeiro, $R J$ - Brazil

\section{Abstract}

Ischemic strokes secondary to occlusion of large vessels have been described in patients with COVID-19. Also, venous thrombosis and pulmonary thromboembolism have been related to the disease. Vascular occlusion may be associated with a prothrombotic state due to COVID-19-related coagulopathy and endotheliopathy. Intracranial hemorrhagic lesions can additionally be seen in these patients. The causative mechanism of hemorrhage could be associated with anticoagulant therapy or factors such as coagulopathy and endotheliopathy. We report on cases of ischemic, thrombotic, and hemorrhagic complications in six patients diagnosed with SARS-CoV-2 infection. Chest computed tomography (CT) showed typical SARS-CoV-2 pneumonia findings in all the cases, which were all confirmed by either serology or reverse transcription polymerase chain reaction (RT-PCR) tests.

\section{Case 1}

A 29-year-old woman who was 5 weeks pregnant, previously healthy, was diagnosed with COVID-1912 days before hospital admission, when both immunoglobulins (IgM and IgG) were positive. While in home quarantine, the patient manifested sudden right hemiplegia and aphasia 48 hours before hospital admission. Upon arrival to the emergency room, she was disoriented and had right hemiplegia.

\section{Keywords}

COVID-19/complications; Pneumonia; Hemorrhage; Thromboembolism/complications; Spectrum Analysis/ methods; Diagnostic Imaging/methods.
Brain magnetic resonance (MR) angiography revealed occlusion at the left internal carotid bifurcation extending to the corresponding middle cerebral artery and A1 segment of the ipsilateral anterior cerebral artery. There were areas of hyperintense signal on fluid-attenuated inversion recovery (FLAIR) sequence and restricted diffusion in the left caudate, internal capsule, putamen, insula, centrum semiovale, and cortical territories of the ipsilateral middle cerebral artery, with mild sulcal effacement, indicating subacute ischemic injury. Susceptibility weighted imaging (SWI) demonstrated hematic material in the left putamen (Figure 1).

Chest CT showed peripheral ground-glass opacities associated with foci of consolidation, typical of COVID-19 pneumonia ${ }^{1}$. Brain CT showed an ischemic lesion with a small hemorrhagic area (Figure 2). The patient was transferred to the intensive care unit for supportive treatment.

\section{Case 2}

A 59-year-old man presented with fever, myalgia, arthralgia, headache, and dyspnea. Because of the patient's clinical worsening status and need for supplemental oxygen, hospitalization was required. RT-PCR testing was positive for SARS-CoV-2 infection. Chest CT showed ground-glass opacities and bilateral consolidations, typical of COVID-19 pneumonia (Figure 3).

After 2 days of hospitalization, the patient had respiratory failure requiring orotracheal intubation. There was an increase in D-dimer level. Chest CT with contrast showed pulmonary thromboembolism in the lingular and left lower lobe branches (Figure 4, arrows). Anticoagulation therapy was implemented.

Mailing Address: Pedro Castro

Avenida Jorge Curi, 550. Postal Code: 22775-001, Barra da Tijuca, Rio de Janeiro, RJ - Brazil.

E-mail:pnpdc@yahoo.com.br 


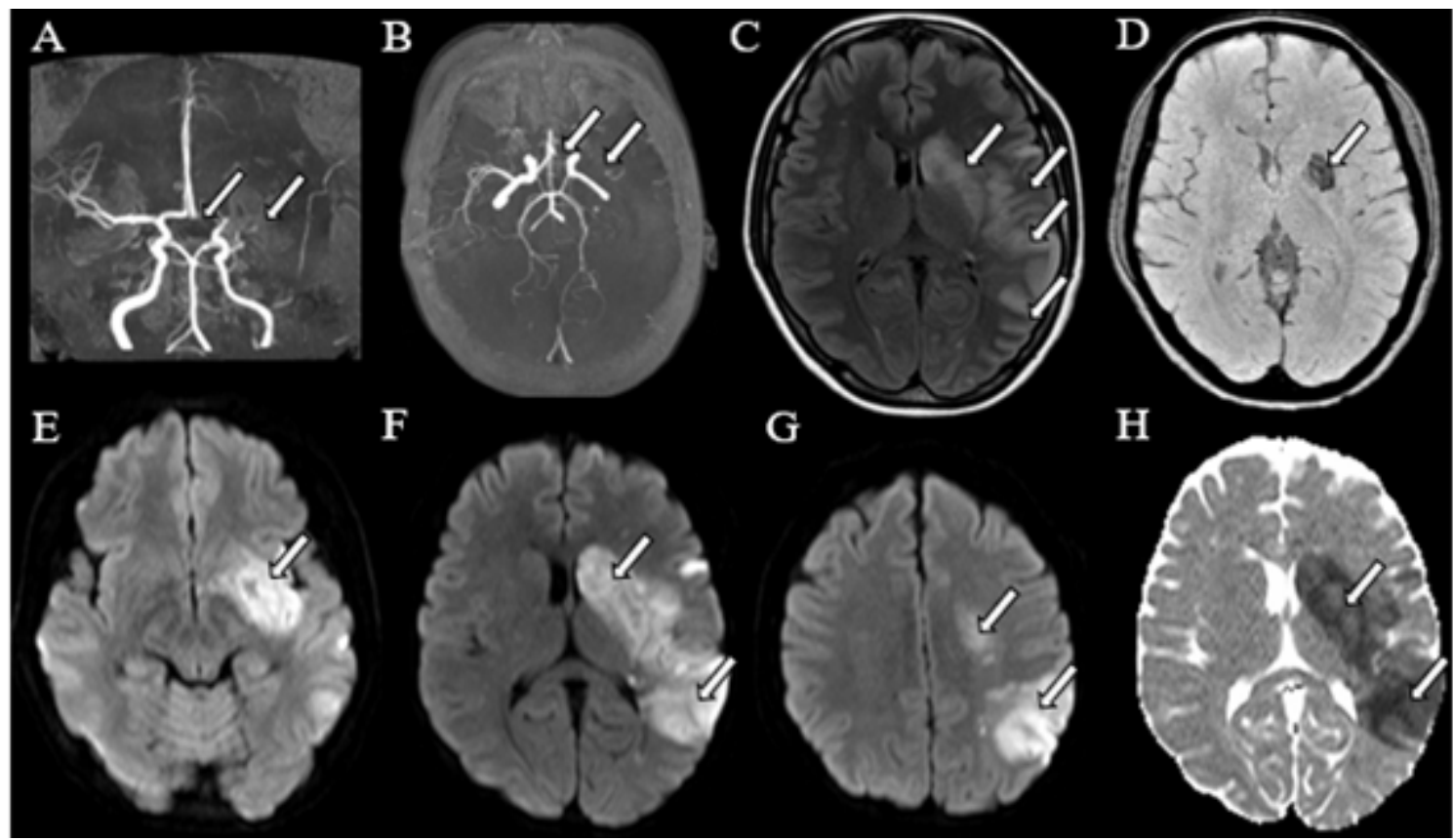

Figure 1 - 3D-TOF MRA (A, B) demonstrates occlusion at the left ICA bifurcation extending to the MCA and ACA stems; FLAIR image (C) shows hyperintensity in left MCA territory, including basal ganglia; SWI (D) hypointensity in left basal ganglia indicating hemorrhagic foci; DWI (E, F,G) and ADC-map demonstrate markedly restricted diffusion, indicating subacute stroke.
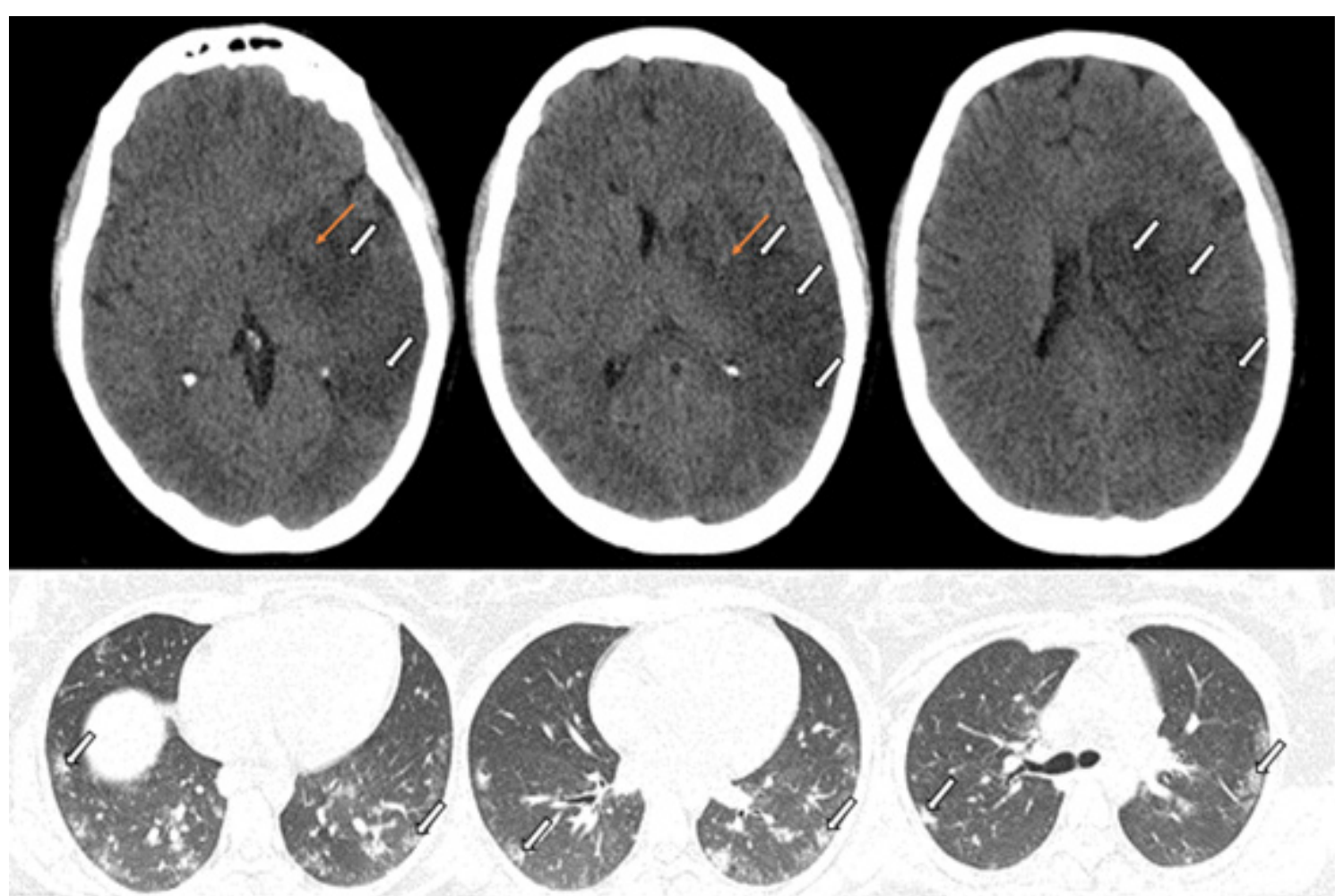

Figure 2 - Unenhanced brain CT scan (top row) demonstrates hypodense areas in MCA territory, including basal ganglia, suggestive of a subacute stroke with subtle hyperdense foci, indicating hemorrhage. Chest CT scan (bottom row) shows bilateral patchy peripheral ground-glass opacities associated with consolidation foci, typical CT imaging features for COVID-19. 


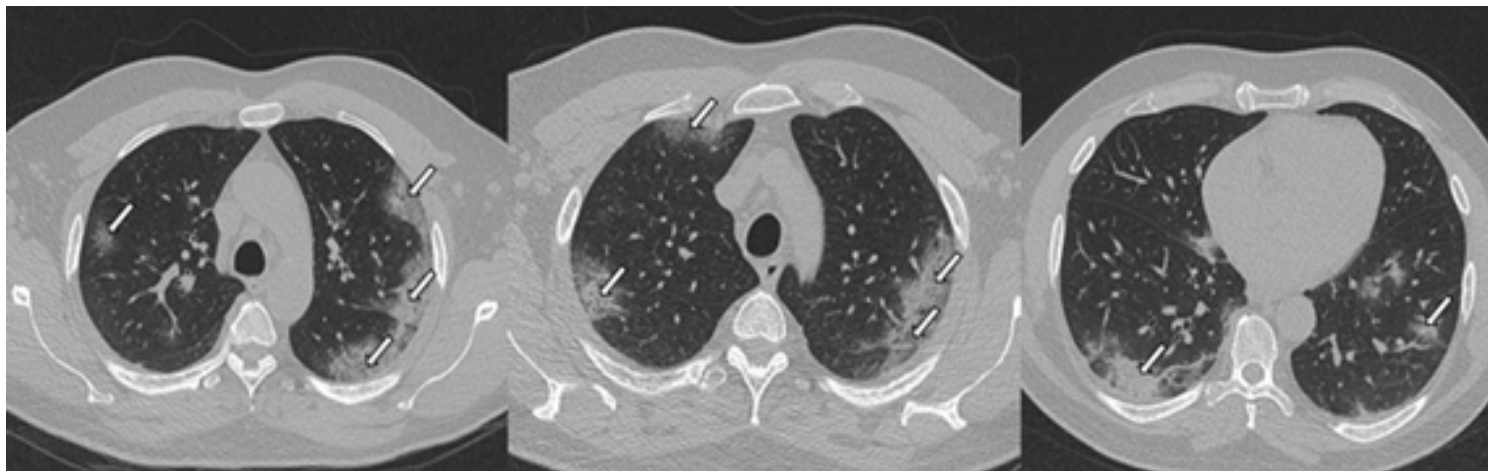

Figue 3 - Chest CT scan shows bilateral, multifocal rounded and peripheral ground-glass opacities with superimposed intralobular septal thickening and consolidation, which are typical features for COVID-19 pneumonia ${ }^{1}$.

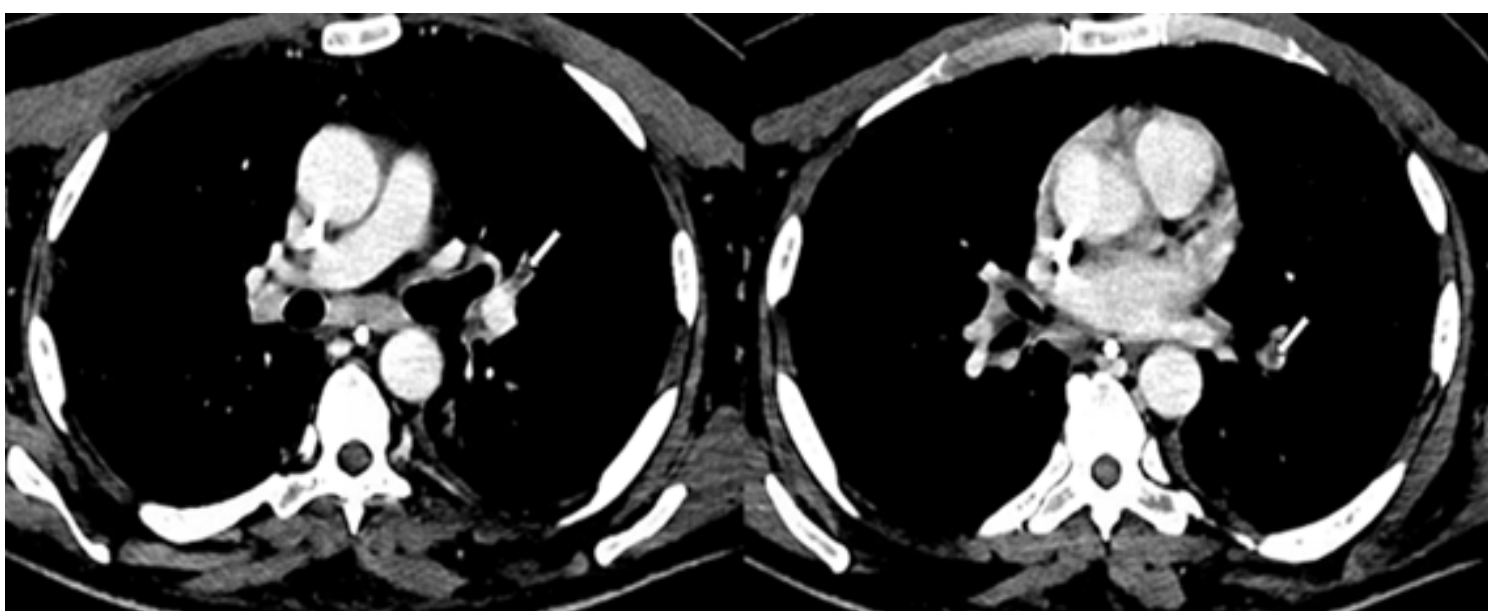

Figure 4-CT pulmonary angiography shows filling defects within the lingular and left lower lobe branches, suggestive of acute pulmonary embolism (arrows).

On the 30th day of hospitalization, the patient had cardiac arrest with asystole, which was reversed after 5 minutes. Brain CT showed massive intraparenchymal hemorrhage in the left frontal region associated with subarachnoid hemorrhage (Figure 5). Surgical evacuation of the hematoma was performed.

Subsequent MRI showed post-surgical changes and subcortical and deep microbleeds in both cerebral hemispheres and corpus callosum, which may be associated with thrombotic microangiopathy (Figure 6). In addition, a FLAIR hyperintense signal was demonstrated in the thalami and caudate nuclei, probably related to a hypoxic-ischemic injury (Figure 7).

\section{Case 3}

A 30-year-old woman presented with severe respiratory failure and reversed cardiac arrest in the emergency room. Chest CT demonstrated groundglass opacities and bilateral peripheral consolidations, typical of COVID-19 pneumonia ${ }^{1}$ (Figure 8). Brain CT demonstrated hypodensity in the cerebral cortex bilaterally, as well as in the putamina and caudate nuclei, indicative of severe hypoxic-ischemic injury (Figure 9). The patient was transferred to the intensive care unit for supportive treatment. RT-PCR testing was positive for SARS-CoV-2 infection. 


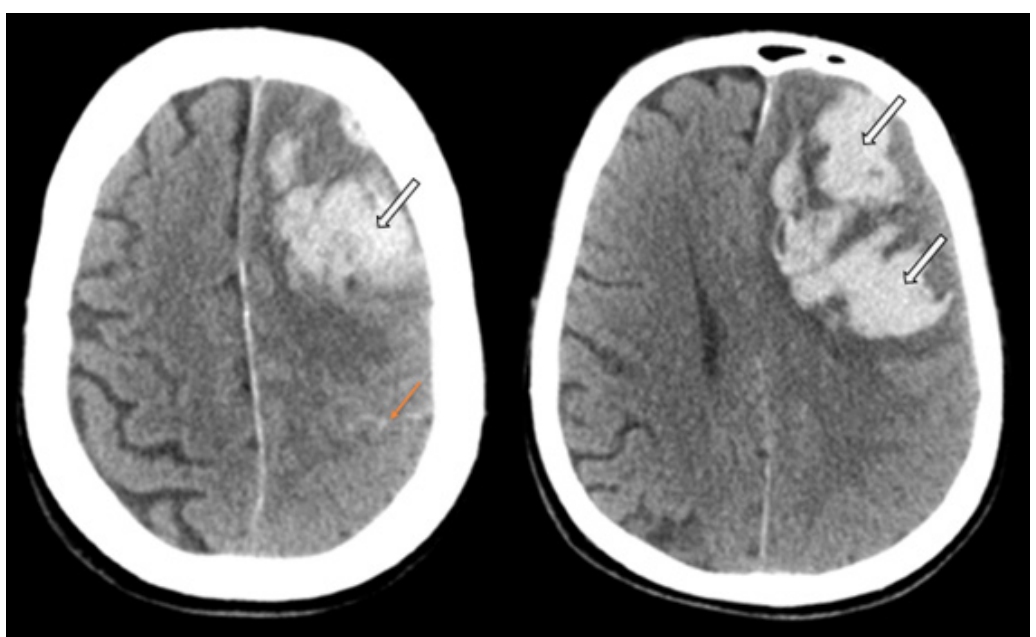

Figure 5 - Unenhanced brain CT scan shows left frontal lobe hematoma and subarachnoid hemorrhage.

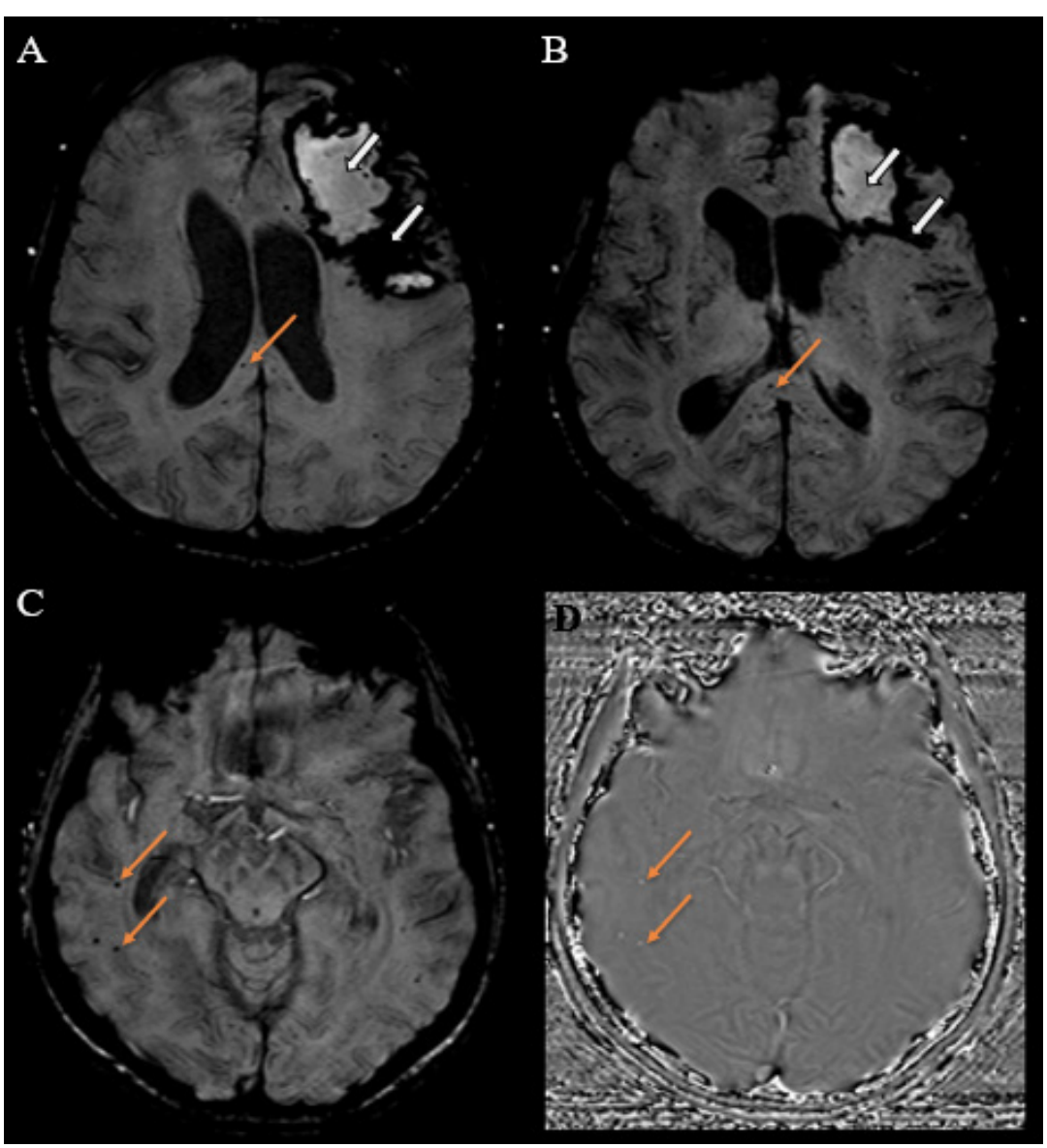

Figure 6 - MRI SWI (a, b, c) and phase-map image (d) demonstrate surgical changes and blood degradation products in left frontal lobe. Note multiple subcortical and deep microbleeds in both cerebral hemispheres and corpus callosum, what may be associated with thrombotic microangiopathy. 


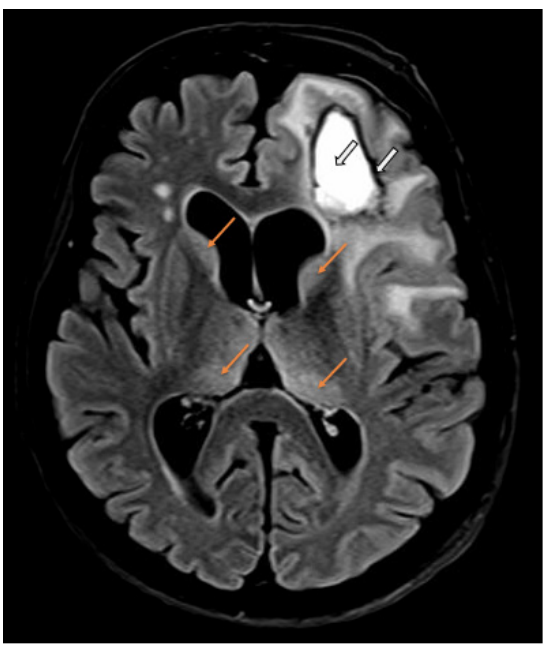

Figure 7 - FLAIR image shows surgical changes and blood degradation products in left frontal lobe and hyperintensity in thalami and caudate nuclei, probably related to hypoxic-ischemic injury.

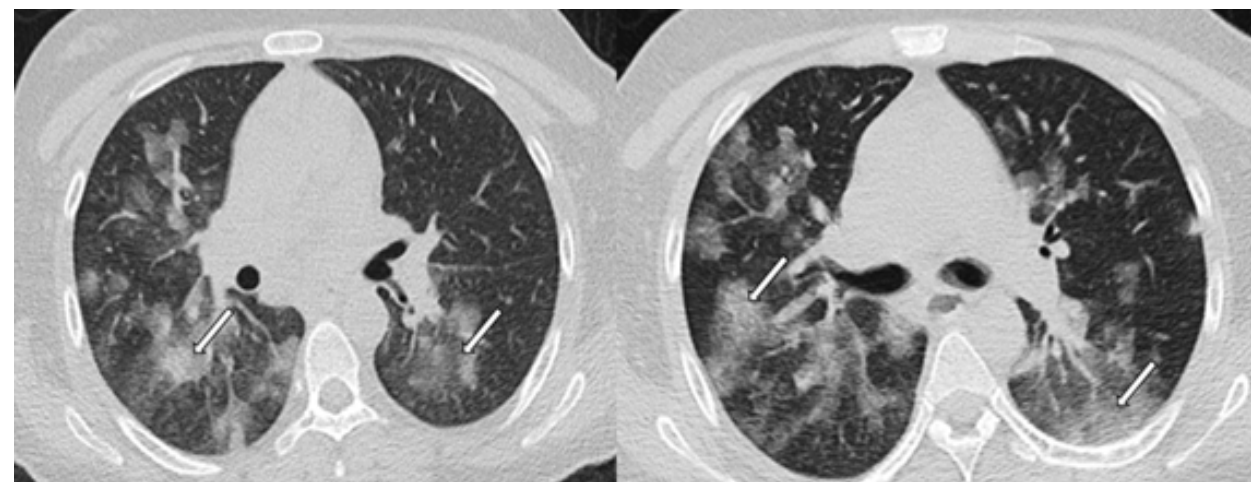

Figure 8 - Chest CT shows bilateral round ground-glass opacities and bilateral patchy consolidations.

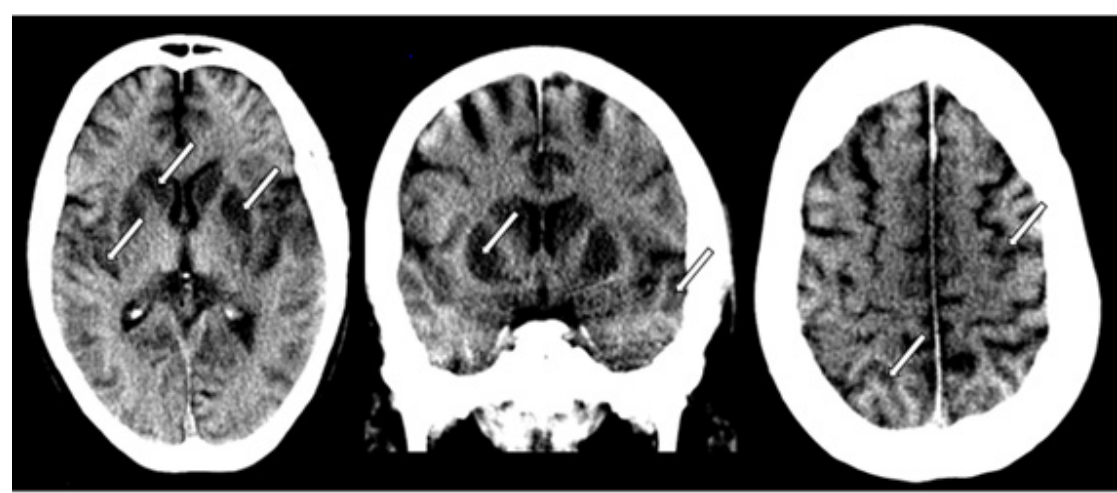

Figure 9 - Brain CT scan demonstrates brain volume loss associated with hypodensity in the cerebral cortex bilaterally, as well as in the putamina and caudate nuclei, indicative of severe hypoxic-ischemic injury. 


\section{Case 4}

A 70-year-old man without comorbidities presented with fever, anosmia, and dyspnea for 4 days. At the emergency department, chest CT demonstrated peripheral ground-glass opacities, crazy-paving pattern, and parenchymal bands (Figure 10). RT-PCR testing was positive for SARS-CoV-2 infection. Upon hospitalization, the patient received full-dose enoxaparin. After 2 days, the patient had a decrease in his level of consciousness. Brain CT demonstrated a large intraparenchymal hematoma in the left frontotemporoparietal region, associated with intraventricular hemorrhage (Figure 11).
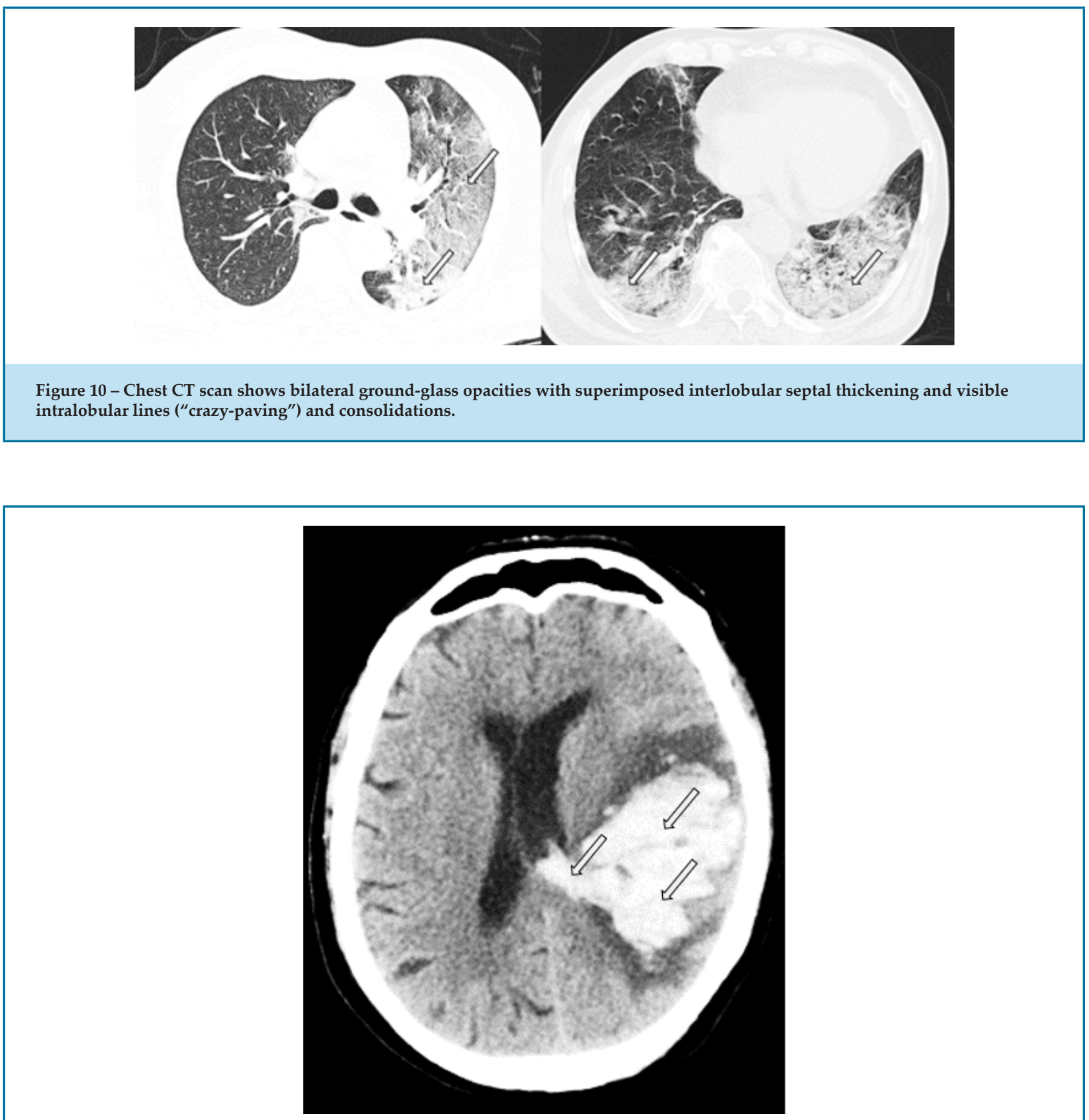

Figure 11 - Brain CT scan demonstrates a large intraparenchymal hematoma in left frontal and parietal lobes, associated with intraventricular hemorrhage. 


\section{Case 5}

A 78-year-old man had hypertension, diabetes, and chronic obstructive disease. After 7 days of flulike symptoms, the patient developed dyspnea and was admitted to the emergency room. CT revealed bilateral ground-glass opacities, typical of COVID-19 pneumonia $^{1}$ (Figure 12). RT-PCR testing was positive for SARS-CoV-2 infection.

The patient was hospitalized for 23 days, during which he was treated with antibiotics and corticosteroids. With the improvement of symptoms, the patient was discharged from hospital. After 7 days at home, he presented with severe respiratory distress and returned to the emergency department. Oxygen saturation was $70 \%$ with an increase to $95 \%$ after oxygen supplementation. There was an increased D-dimer level.

Chest $\mathrm{CT}$ angiography showed a filling defect in the right posterior basal segment branch, compatible with pulmonary thromboembolism (Figure 13). In addition, there was a deterioration in the pulmonary ground-glass opacities, with more than $50 \%$ of the lungs compromised (Figure 14). Doppler ultrasonography of left upper limb demonstrated thrombosis of the left cephalic vein. The patient underwent orotracheal intubation and was transferred to the intensive care unit.

\section{Case 6}

A 45-year-old man had flu-like symptoms and diarrhea for 2 days. Chest tomography performed in the emergency room showed a typical pattern for COVID-19 pneumonia, with pulmonary involvement estimated at less than $25 \%$ by visual analysis ${ }^{1}$ (Figure 15, A). The patient was discharged from hospital with prescribed cefuroxime and azithromycin and reported improvement in fever and diarrhea during antibiotic therapy.

After 10 days of discharge, the patient returned to the emergency room with severe abdominal pain, refractory to medication, and desaturation of $89 \%$, without respiratory distress. Chest CT showed significant worsening of pulmonary findings, with an estimated involvement of more than 50\% (Fig 15, B). Laboratory tests revealed a significant increase in D-dimer level, slightly increased lactate, and elevated urea and creatinine.

On the 4th day of hospitalization in the intensive care unit, while using $1 \mathrm{mg} / \mathrm{kg}$ of enoxaparin twice daily, the patient developed leukocytosis, pain, and abdominal distension without signs of peritoneal irritation. He underwent CT angiography of the chest, abdomen, and pelvis, which revealed thrombosis in the proximal portion of the superior mesenteric artery and branch (Figure 16).
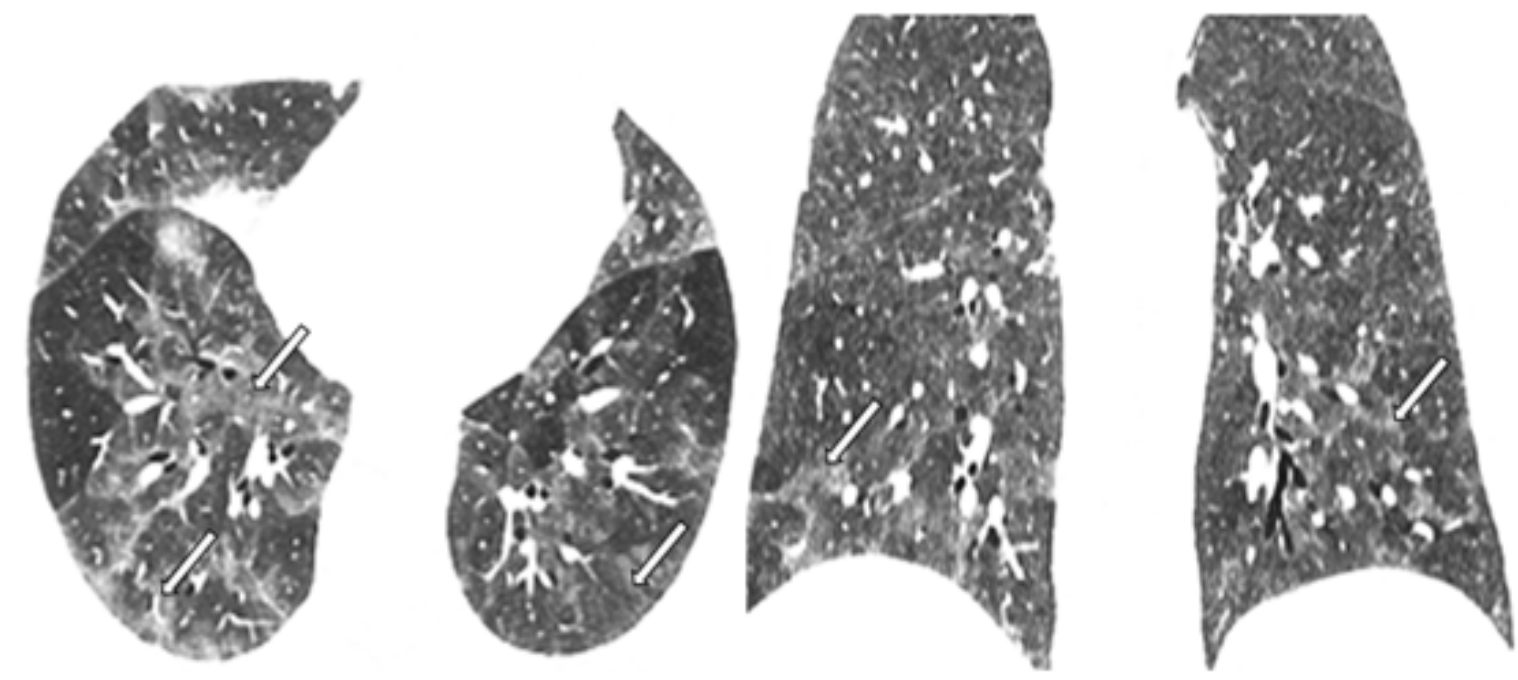

Figure 12 - CT scan reveals bilateral ground-glass opacities, typical of Covid-19 pneumonia ${ }^{1}$. 


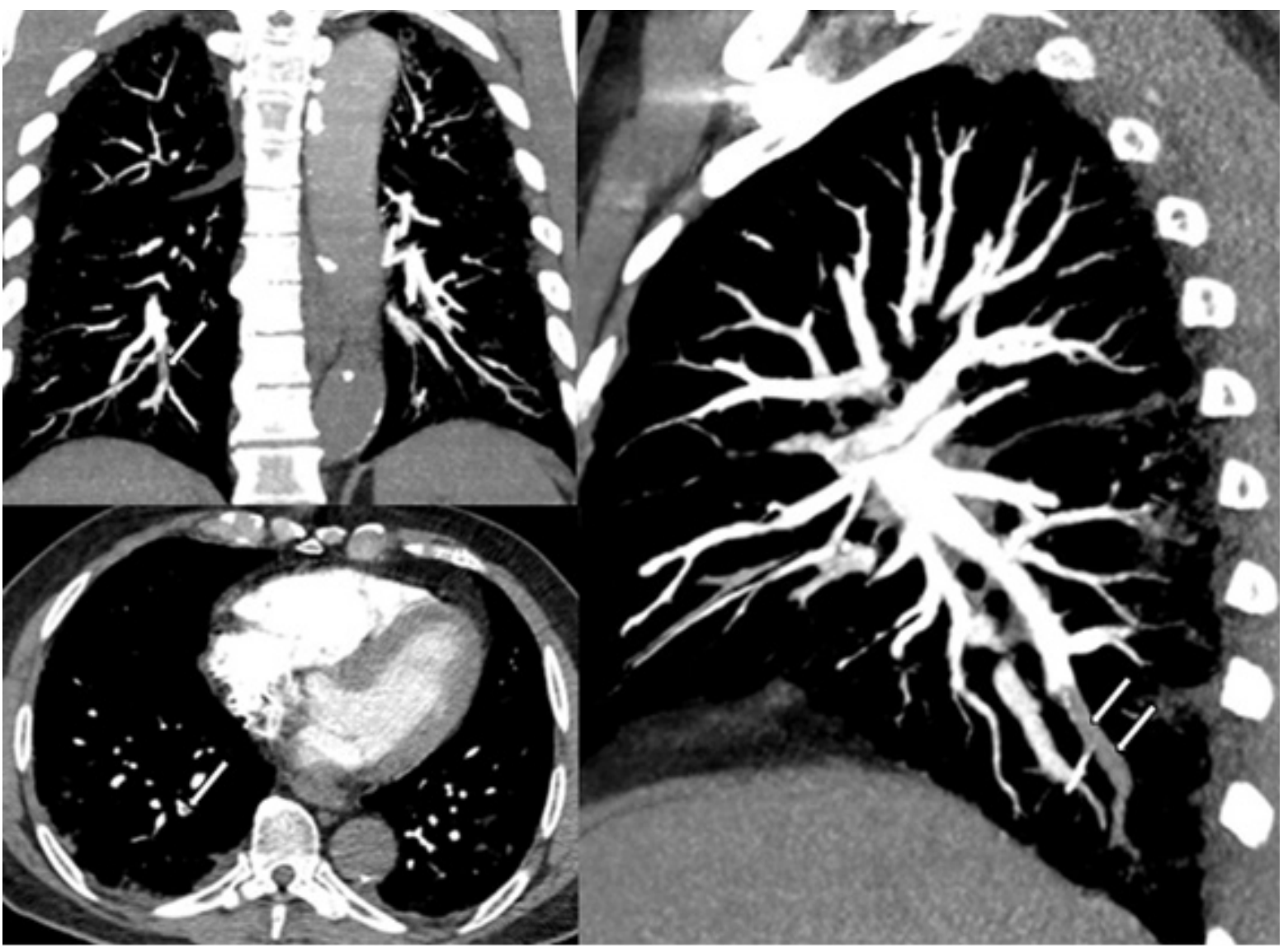

Figue 13 - CT pulmonary angiography shows filling defects within right posterior basal segment branch, suggestive of acute pulmonary embolism.

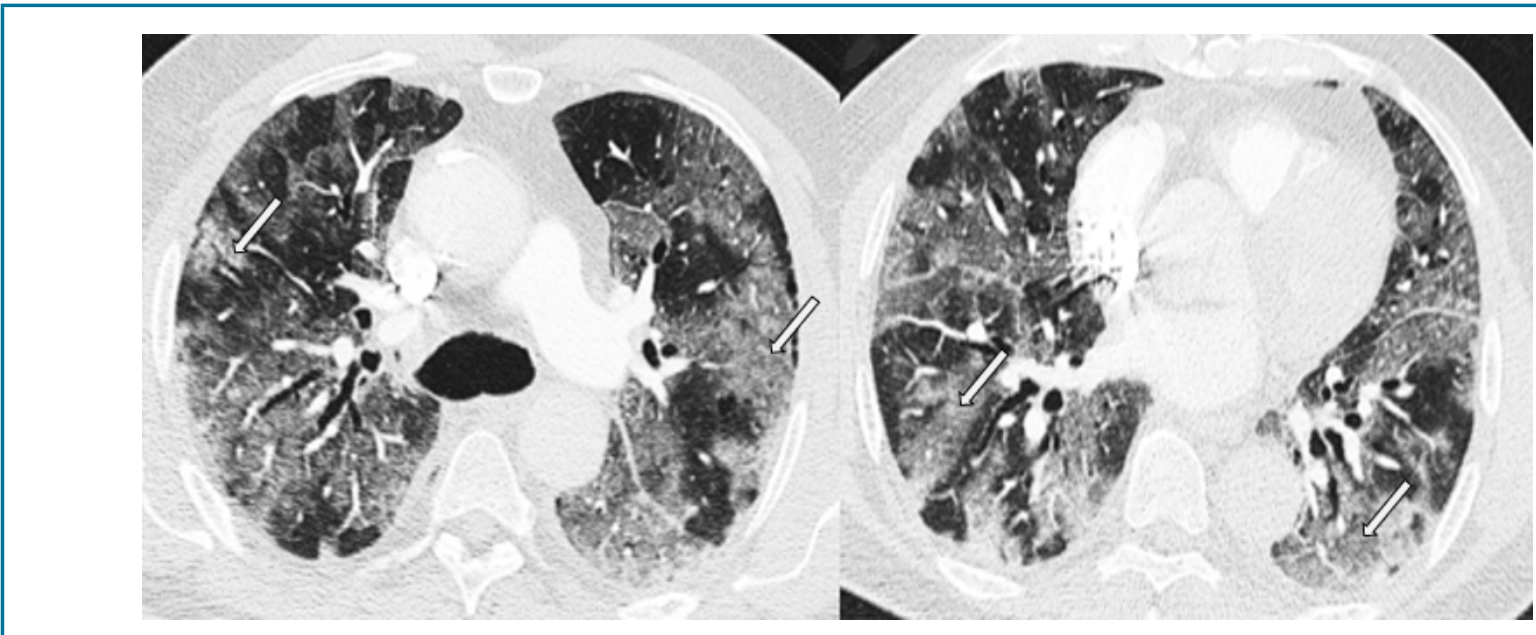

Figure 14 - CT scan reveals worsening of the pulmonary bilateral ground-glass opacities, now associated with superimposed interlobular septal thickening and intralobular lines. 


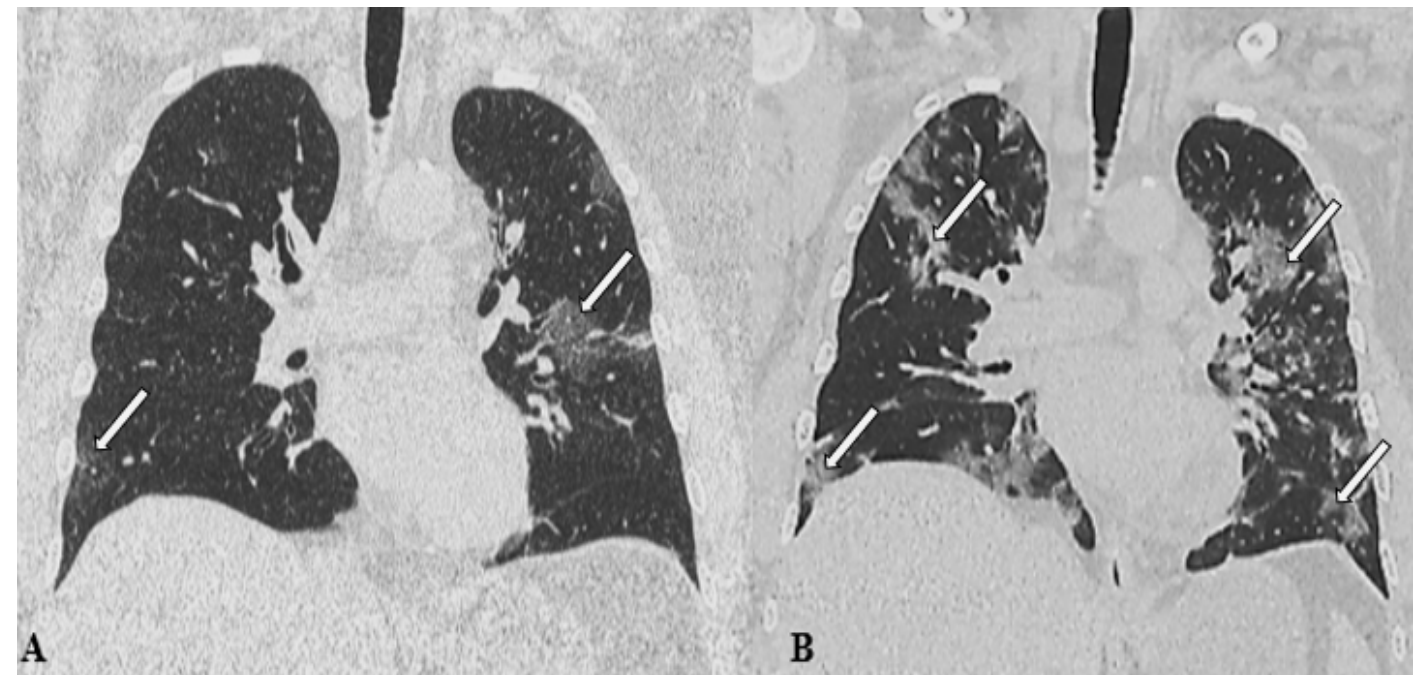

Figure 15 - Chest CT on the first emergency care, revealing typical findings of Covid-19 pneumonia (A). Examination after 10 days reveals worsening of the findings (B).

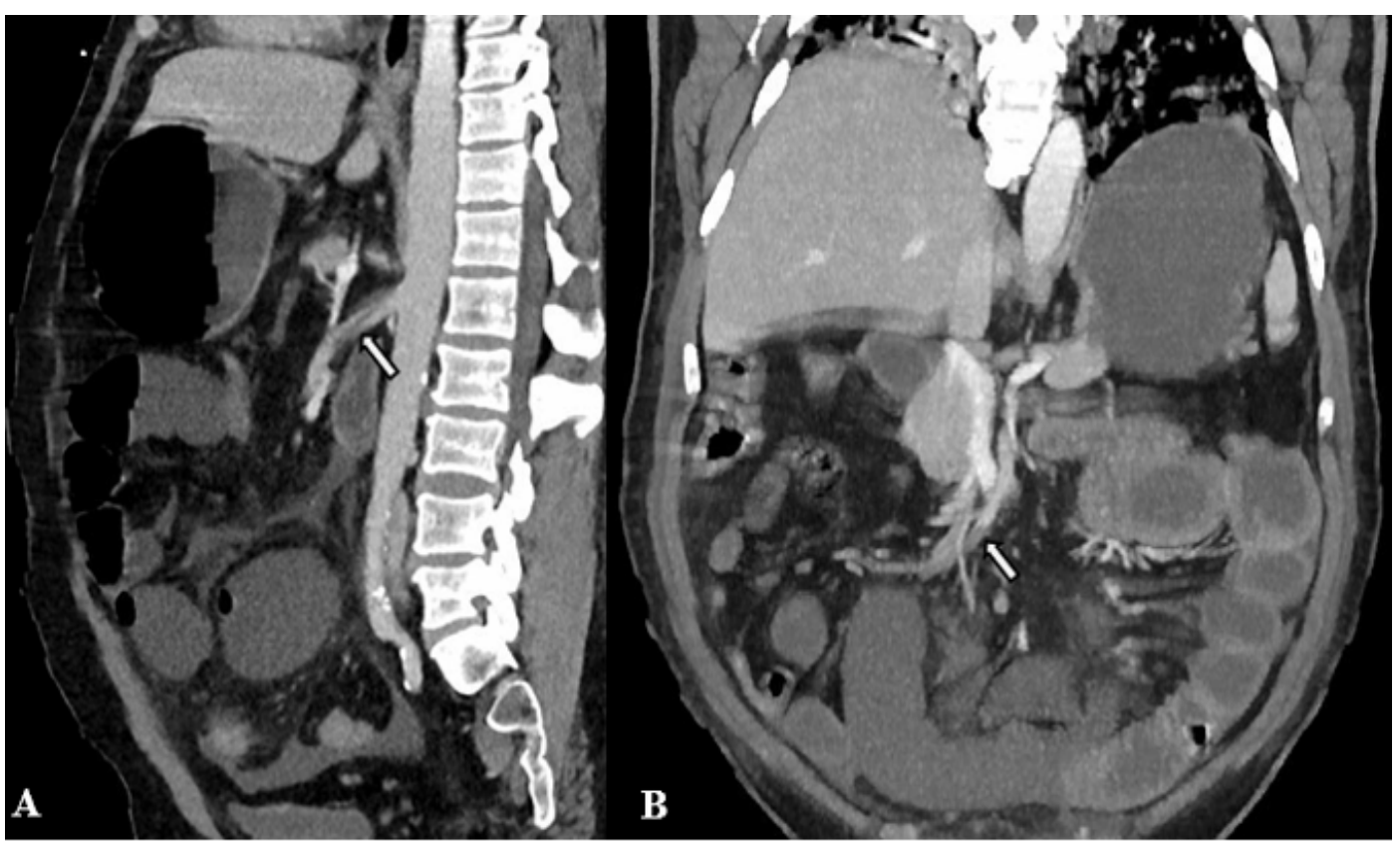

Figure 16 - Angiotomography 2 days after admission, revealing a thrombus in the upper mesenteric artery (A, arrow). MIP reformatation demonstrates thrombosis in one of its branches (B, arrow). 
Upon diagnosis, the patient underwent mechanical thrombectomy and intra-arterial thrombolysis in occluded branches as well as angioplasty with stenting in the proximal third of the superior mesenteric artery. There was partial reperfusion of the branches occluded at the end of the procedure.

\section{Discussion}

SARS-CoV-2 emerged in December 2019 in Wuhan, China, and has since spread worldwide. ${ }^{2}$ The World Health Organization declared the coronavirus outbreak a pandemic in March 2020. ${ }^{3}$ The severe forms of COVID-19 manifest mainly as acute pulmonary respiratory syndrome. However, the involvement of other systems, such as the gastrointestinal and nervous systems, has also been demonstrated. ${ }^{4}$ The involvement of the central nervous system may include encephalitis, myelitis, and cerebrovascular disease. ${ }^{5-8}$

The patterns of brain ischemia in patients with COVID-19 may include large vessel occlusion with territorial infarct and hypoxic-ischemic injury related to hypoxemia or cardiopulmonary arrest. ${ }^{9}$ Severe hypoxic-ischemic injury manifests in regions of greater metabolic demand, such as the cortex, basal ganglia, and thalamus. ${ }^{10}$ The patient in case 2 presented with lesions in the thalamus and caudate. In case 3 , there were lesions in the cortex, putamen, and caudate. The presence of severe hypoxic-ischemic injury may be implicated in a longer hospital stay, larger neurological sequelae, and a dismal prognosis.

Also, an increase in the incidence of ischemic strokes secondary to occlusion of large vessels has been reported in patients infected with SARS-CoV-2. ${ }^{7}$ Case 1 demonstrates a young patient, without cardiovascular risk factors or other comorbidities, presenting with ischemic stroke due to occlusion of large vessels during the course of COVID-19. Case 6 demonstrates thrombosis in the superior mesenteric artery and branch. Likewise, a rising prevalence of venous thrombosis and pulmonary thromboembolism has been related to the disease. ${ }^{8}$ Cases 2 and 5 demonstrate this form of vascular complication.

Vascular occlusion could be connected to a prothrombotic state related to angiotensin-converting enzyme 2 (ACE2) downregulation and inflammationinduced coagulopathy in COVID-19. Additionally, changes in coagulation with increased D-dimer level and fibrin/fibrinogen degradation products may be seen in these patients. ${ }^{11}$ Such abnormalities may be related to a higher incidence of thrombotic events, including ischemic stroke related to large vessel occlusions, as well as pulmonary thromboembolism related to venous thrombosis.

The vascular endothelium is an extensive and active paracrine, endocrine, and autocrine organ that has an important role in controlling vascular tone and homoeostasis. ${ }^{12}$ When this hemostatic balance is disturbed by a viral infection eliciting endothelial dysfunction, it produces vasoconstriction, hypoxia, and acute vascular wall inflammation ${ }^{13}$. Apoptosis of the endothelial cells leads to exposure of the intima (smooth muscle cells, extracellular matrix, collagen 1) generating platelet activation (hypercoagulability) and activation of innate and adaptable immunological responses (inflammation in situ and microvasculature dysfunction). ${ }^{13}$

Endotheliopathy, disseminated intravascular coagulation, and sepsis-associated coagulopathy may be related to multiple organ failure in critically ill patients, including those with COVID-19. ${ }^{11}$ Thrombotic microangiopathies, such as disseminated intravascular coagulation, can manifest as diffuse cerebral microbleeds. ${ }^{14}$ The patient in case 2 exhibited cortico-subcortical and deep microbleeds, including the corpus callosum, possibly related to this pattern of vasculopathy. ${ }^{15}$ However, factors such as hypoxia and endotheliopathy may be implicated somehow. ${ }^{15}$

Intracranial hemorrhagic lesions can also be seen in these patients. ${ }^{4,8,9}$ The causative mechanism of hemorrhage can be mainly associated with clinically indicated anticoagulant therapy; however, it is not clear whether factors such as coagulopathy and COVID-19related endotheliopathy may be involved in some way. Cases 2 and 4 demonstrate large intraparenchymal hematomas in patients treated with anticoagulation during SARS-CoV-2 infection.

Finally, the vascular complications that may occur during COVID-19 are of great therapeutic importance. Cerebral infarction related to thrombosis of large vessels requires the implementation of immediate recanalization therapy, if the patient is eligible. In addition, other approaches that may still be required are 
full anticoagulation for pulmonary thromboembolism as well as surgical evacuation of intracranial hematomas. The medical team must be aware of the presence of coagulopathy and a prothrombotic state in these patients, which may be related to the higher incidence of some of these complications and directly interfere with the treatment.

\section{Author contributions}

Conception and design of the research: Castro $\mathrm{P}$, Chagas L, Machado D. Writing of the manuscript : Castro P, Chagas L, Wajnberg E. Critical revision of the manuscript for intellectual content : Cougo P, Santos R, Machado D.

\section{References}

1. Simpson S, Kay F, Abbara S, Bhalla S, Chung J, Chung M, et al. Radiological Society of North America Expert Consensus Statement on Reporting Chest CT Findings Related to COVID-19. Endorsed by the Society of Thoracic Radiology, the American College of Radiology, and RSNA. J Thorac Imaging.2020;35(4):219-27. https://doi.org/10.1148/ ryct.2020200152

2. Wu Y.Xu X, Chen Z, Duan J, Hashimoto K, Yang L, et al. Nervous system involvement after infection with COVID-19 and other coronaviruses. Brain Behav Immun. 2020;87:18-22.

3. Jin H, Hong C, Chen S, Zhou Y, Wang Y, Mao L. Consensus for prevention and management of coronavirus disease 2019 (COVID-19) for neurologists. Stroke Vasc Neurol. 2020;5(2):146-51. doi: 10.1136/svn2020- 000382

4. Sharifi-Razavi A, Karimi N, Rouhani N. COVID 19 and Intra cerebral hemorrhage: causative or coincidental. New Microb New Infect. 2020; 35:100669.

5. Hayashi M. COVID-19-associated mild encephalitis/encephalopathy with a reversible splenial lesion.J Neurol Sci .2020;415:116941.

6. Zhao K.Acute myelitis after SARS-CoV-2 infection: a case report. MedRxiv 2020 april 9 [Epub ahead of print]. doi10.1101/2020.03.16.20035105. ???

7. Oxley TJ. Large-Vessel Stroke as a Presenting Feature of Covid-19 in the Young. N Engl J Med.2020;382(20): e60.

\section{Potential Conflict of Interest}

No potential conflict of interest relevant to this article was reported.

\section{Sources of Funding}

There were no external funding sources for this study.

\section{Study Association}

This study is not associated with any thesis or dissertation work.

\section{Ethics approval and consent to participate}

This article does not contain any studies with human participants or animals performed by any of the authors.

8. Divani AA, Andalib S, Di Napoli M, Lattanzi S, Hussain MS, et al Coronavirus Disease 2019 and Stroke: Clinical Manifestations and Pathophysiological Insights. J Stroke Cerebrovasc Dis.2020;29(8):104941 Volume 29, Issue 8, August 2020.

9. Parry AH, Wani AH, Yaseen M. Neurological Dysfunction in Coronavirus Disease-19 (COVID-19). Acad Radiol. 2020;27(9):1329-30.

10. Huang BY, Castillo M. Hypoxic-ischemic brain injury: imaging findings from birth to adulthood. Radiographics. 28 (2): 417-39. doi:10.1148/ rg.282075066

11. Connors JM, Levy JH. COVID-19 and its implications for thrombosis and anticoagulation. Blood. 2020; 135(23):2033-40.

12. Flammer AJ, Anderson T, Celermajer DS, Creager MA, Deanfield J, et al. The assessment of endothelial function: from research into clinical practice. Circulation. 2012; 126(6): 753-67.

13. Bonetti PO, Lerman LO, Lerman A. Endothelial dysfunction - a marker of atherosclerotic risk. Arterioscl Throm Vas. 2003;23(2):168-75.

14. Osborn AG. Vasculopathy. In: Osborn AG, Hedlund GL, Salzman KL, Osborn's Brain. 2nd ed Philadelphia: Elsevier, 2017.

15. Fitsiori A, Pugin D, Thieffry C, Lalive P,Vargas MI. (2020). Unusual Microbleeds in Brain MRI of Covid-19 Patients. J Neuroimaging. 2020;30(5):593-7. https://doi.org/10.1111/jon.12755. 